\title{
The development of individual cognitive stimulation therapy (iCST) for dementia
}

This article was published in the following Dove Press journal:

Clinical Interventions in Aging

30 December 2014

Number of times this article has been viewed

\section{Lauren A Yates ${ }^{1,2}$ \\ Phuong Leung ${ }^{2}$ \\ Vasiliki Orgeta ${ }^{2}$ \\ Aimee Spector ${ }^{1,3}$ \\ Martin Orrell ${ }^{1,2}$}

'North East London Foundation Trust, ${ }^{2}$ Division of Psychiatry, University College London, ${ }^{3}$ Research Department of Clinical, Educational and Health Psychology, University College London, London, UK
Correspondence: Lauren A Yates Division of Psychiatry, University College London, Ist Floor Charles Bell House, 67-73 Riding House Street, London, WIW 7EJ, UK

Email lauren.yates@ucl.ac.uk
Background: Adopting a systematic approach to the development of an intervention, supported by robust theoretical, empirical, and clinical rationales represents best practice. The Medical Research Council (MRC) provides a framework for a systematic step-wise approach to the evaluation of complex interventions. This study describes the development phase of the individual cognitive stimulation therapy (iCST) for dementia trial, within this framework.

Methods: In the preclinical phase, a recent Cochrane Review of cognitive stimulation for dementia and the current literature on individual cognitive stimulation interventions were examined to establish an evidence base. In addition, people with dementia, carers, and care staff were consulted regarding the acceptability of iCST, and a panel was put together to advise the team on the adaptation of group cognitive stimulation therapy (CST). Phase I (modeling) involved consultations with service users and experts in a series of focus groups, interviews, an online survey, and a consensus conference. Finally, Phase II field testing of the intervention was carried out.

Results: Two drafts of the materials were produced before a final version ready for use in the main randomized controlled trial (RCT). Key changes between the drafts included: editorial amendments to improve the clarity of instructions, emphasize the person centeredness of the approach, and reduce the overall length of the introduction section; the simplification of academic terminology and activities deemed "too difficult"; adjustments made to the monitoring-progress forms and session rating scale to enhance user-friendliness; the addition of a "Getting started" section; amendments made to the content of the toolkit; and clearer distinction made between the level of difficulty of activities.

Conclusion: The rigorous development of the intervention was beneficial as the feasibility of the intervention was explored both in theory and practice, and consulting with service users ensured that materials were appropriately tailored to their needs. A Phase III RCT is currently being conducted to determine the effectiveness of iCST.

Keywords: cognitive stimulation, Delphi consensus, MRC framework, intervention development, intervention evaluation

\section{Introduction}

Complex interventions such as psychological therapies are used in health and social services. ${ }^{1}$ In order for policy makers to justify their implementation, they must be shown to be cost-effective and have an evidence base. The application of a rigorous development and evaluation strategy is advantageous, as the resulting complex intervention is more likely to be well designed; founded in robust theoretical, empirical, and clinical rationales; and developed enough to be expected to have a worthwhile effect. "Cognitive stimulation therapy" (CST) is an evidence-based psychosocial intervention for people with mild to moderate dementia, which has been shown to benefit cognition and quality of life (QoL). ${ }^{3,4}$ As well as comparing favorably to trials 
of anticholinesterase inhibitors, economic analysis of CST has demonstrated its cost-effectiveness. ${ }^{5,6}$ As a result, the intervention is recommended by the UK's National Institute for Health and Care Excellence (NICE), and has been the focus of reports published by the National Health Service (NHS) Institute for Innovation and Improvement in Britain, and Alzheimer's Disease International. ${ }^{6-8}$

Following the encouraging findings of the original CST program, further research focused on the maintenance of benefits, implementation in practice, and cultural adaptation. ${ }^{9-13}$ Whilst we now have robust evidence for group CST, both short and longer term, there is a clinical need for CST to be adapted so that it can be delivered individually for people with dementia by a friend or family member. Individual cognitive stimulation therapy (iCST) will cater for the needs of those unable or unwilling to access groups due to local service constraints, personal preference (eg, if a person does not like to go to the day center or does not wish to participate in a group environment), or health or mobility problems (eg, housebound, frail, or unable to walk). The effectiveness of iCST will be examined in an innovative clinical trial following the Medical Research Council (MRC) guidelines. ${ }^{1}$

This paper describes the methods and outcomes of the development phase of the trial, which encompassed: identifying the evidence for CST and individual cognitive stimulation, identifying the theories that may explain why these interventions yield benefits, a qualitative modeling process (focus groups, interviews, consensus methods), and field testing the intervention (Figure 1).

\section{Aims and objectives Preliminary development phase}

The aims of the preliminary development phase were: to identify the strengths and limitations of the existing research into individual programs; to develop a theoretical understanding of the mechanisms of action behind the reported benefits of cognitive stimulation and whether these could be applied to iCST; to assess the acceptability of an individualized version of CST suitable for delivery by carers; and to develop the first draft of the program materials, including a manual (Figure 1).

\section{Modeling phase}

The objectives of the modeling process were: to ensure the therapeutic materials were easy to use, clear, and appropriately tailored to the needs of people with dementia and their carers, and to assess the feasibility of the program in theory.

\section{Field-testing phase}

The aims of the field-testing phase were: to evaluate each of the 75 sessions of the program, to determine whether the feasibility concerns highlighted in the focus groups and interviews were speculative or whether they would be occur and act as barriers in practice, and to produce a second draft of the materials.

\section{Consensus process}

The aims of the online survey and consensus conference were: to consolidate the information gathered from the focus groups, interviews, and field testing; to reach consensus on the key themes identified in the analysis of these activities; and to produce the final drafts of the materials.

\section{Stage I: preliminary development of the iCST program Identifying the evidence base (theoretical phase)}

The benefits of CST have been consistently found in both quantitative and qualitative evaluations of CST., ${ }^{4,14} \mathrm{~A}$ recent Cochrane Review suggested systematic evaluation of different modalities of CST, including carer-led versions of the therapy. ${ }^{4}$ There is some evidence to suggest that individual cognitive stimulation can benefit cognitive functioning. A carer-led, home-based program of active training in memory management including cognitive stimulation, orientation, and counseling with psycho-educative elements had long-term benefits (at 18 months' follow-up) for cognition in the person with dementia, reduced care-home admissions, and improved carer well-being. ${ }^{15}$ Although the findings were promising, the sample size of the study was modest, and participants were not randomly distributed between the intervention and control groups. Furthermore, due to the multifaceted nature of the intervention, it was not possible to determine which aspect contributed to the impact on cognition, though the authors posited that this was likely to be explained by the memory management element. The iCST trial will evaluate a program of cognitive stimulation alone, thus the impact on cognition will be more directly measurable. Quayhagen and Quayhagen also found that home-based cognitive stimulation can have a positive impact on both carers and people with dementia. ${ }^{16}$ People with dementia showed improvements in problem solving and memory, and carers a reduction in depressive symptoms.

The work of Onder et al was particularly informative in the development of the design of the iCST program. ${ }^{17}$ Onder 


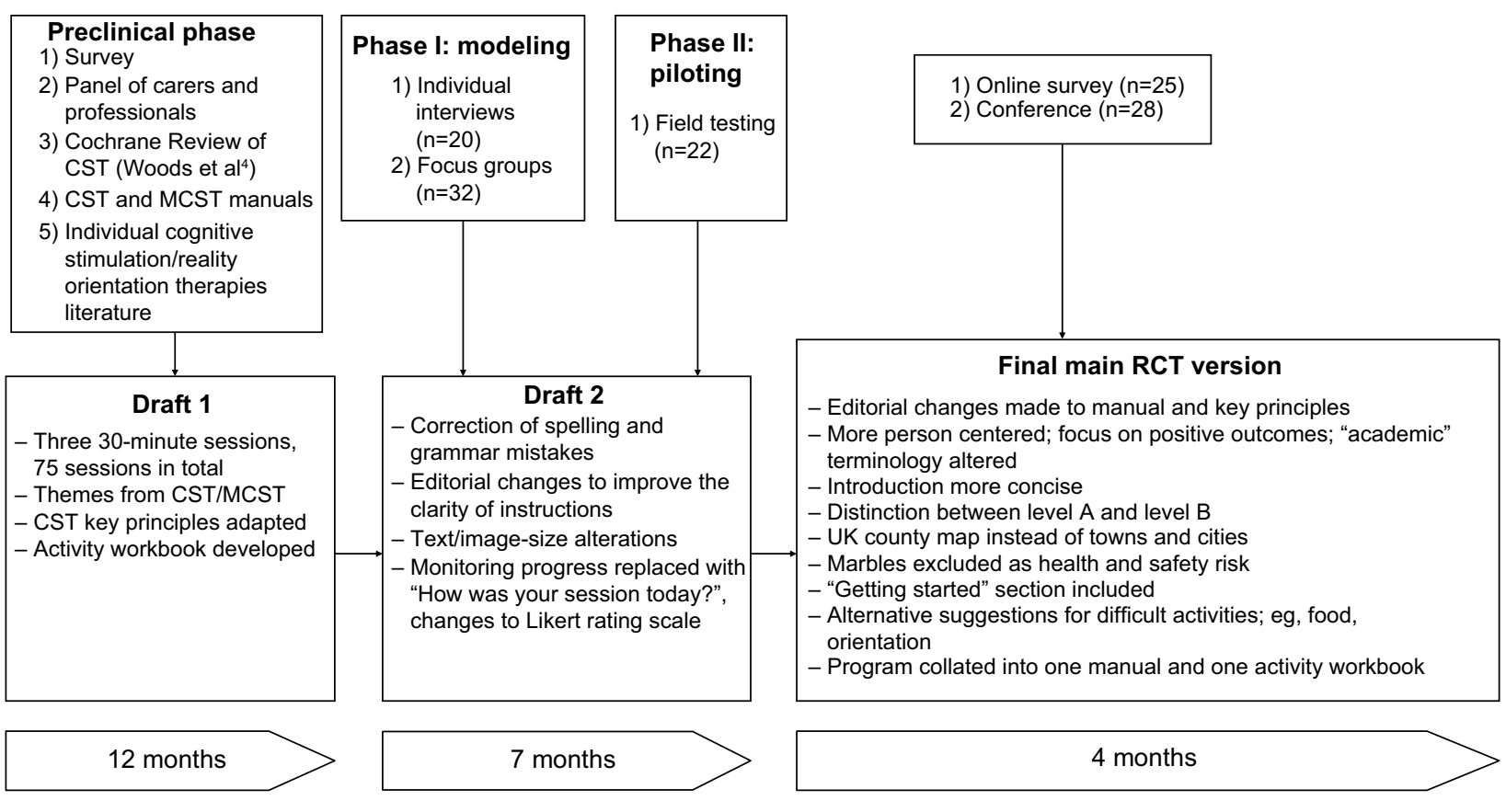

Figure I Development of the individual cognitive stimulation therapy program within the Medical Research Council framework.

Abbreviations: CST, cognitive stimulation therapy; MCST, maintenance cognitive stimulation therapy; RCT, randomized controlled trial.

and colleagues trained family carers to deliver a homebased package of reality orientation and CST. The 25-week program was manualized, with specific schedules for each session. Carers delivered three 30-minute sessions per week. Dyads participating in the program improved relative to the control both in the Mini-Mental State Examination (difference of 1.3 points) and on the Alzheimer's Disease Assessment Scale - cognitive subscale (difference of 2.9 points). A limitation of the study is that adherence to the program was not recorded, thus the intervention may not have been administered according to the study protocol. Informed by this drawback, adherence measures have been designed for use in the iCST trial to maximize treatment fidelity.

Taken together, the Cochrane Review of group CST and studies of individual cognitive stimulation form a substantial evidence base from which the iCST intervention was developed, demonstrating the potential for carers to have an active role in the intervention, and indicating likely outcomes, such as improvements in cognition and QoL for the person with dementia, and well-being for the carer.

\section{Identifying the theory: why might CST and iCST be beneficial?}

The outcomes of CST research and studies exploring individual cognitive stimulation may be understood in the context of the biopsychosocial model of dementia, which describes how psychosocial and biological factors interact to contribute to, and influence outcomes during the course of dementia. ${ }^{18}$ These factors may be fixed and impervious to change, or malleable and susceptible to change and modification (tractable). Cognitive stimulation is identified in the model as a psychosocial intervention that can modify tractable factors, such as mental activity, social psychology, and personal psychology.

\section{Mental stimulation: why does cognitive stimulation benefit cognition?}

Dementia can affect both the episodic and semantic subsystems of explicit memory. In terms of episodic memory, people with dementia often have difficulty acquiring and retaining new information. ${ }^{19}$ However, there is evidence to suggest that capacity for cognitive information processing is not entirely lost. ${ }^{20}$ The iCST program activities have been designed to incorporate implicit learning techniques, stimulate a range of cognitive skills, and encourage the generation of ideas and discussion based on the merits of these elements as observed in group $\mathrm{CST}^{3}$ and the research of Onder et al. ${ }^{17}$

Recently, Hall et al explored the impact stimulating activities have on cognition from a neuropsychological perspective. ${ }^{21}$ In line with the theory of "use it or lose it", ${ }^{22}$ participating in cognitive stimulation may activate neurons, 
which can in turn improve and have a protective effect on their functioning. Cognitive activities may also directly stimulate neuronal systems, enhancing neural pathways responsible for cognitive functions, such as memory and language. Similarly, there is evidence to suggest that social interaction can have a protective effect on cognition. ${ }^{23-25}$ "Social interaction" may constitute an exchange between two or more individuals. The type of interactions a person engages in may depend on personal preference. Moyle et al point out that many family carers assume that day-center attendance or participation in groups is the best "antidote" to or "preventative measure" against social isolation and loneliness without taking into account the person's premorbid personality. ${ }^{26}$ For those who do not enjoy, or find it difficult to participate in a busy group environment due to sensory or cognitive impairment, a oneto-one approach may provide a better quality of engagement and therefore have a more effective impact on cognition and QoL. The delivery of an intervention by someone familiar to the person with dementia may also be advantageous. ${ }^{26}$

\section{Social and personal psychology: why does cognitive stimulation improve QoL?}

Participating in cognitive stimulation has also been shown to yield improvements in QoL. 3,14,27 These gains are thought to be mediated by improvements in cognitive function. ${ }^{27}$ In a qualitative study of the experience of CST, people with dementia described how the groups increased their confidence, and made them feel more positive and relaxed. Alongside the perceived impact on their well-being, they reported improvements in cognitive skills, including memory and concentration, which corroborated proxy observations by CST group facilitators. ${ }^{14}$ Onder et al also noted that participants' increased ability to retain information combined with encouragement from the carer delivering the intervention may have improved their sense of self-esteem. ${ }^{17}$

The person-centered values at the core of CST and iCST may be a mechanism for improvements in QoL. Kitwood developed the conceptual structure of the "malignant social psychology" of dementia, noticing that reductionist biomedical views exacerbated neurological impairment and failed to acknowledge personal experiences of well-being, dignity, and worth. ${ }^{28}$ In response to this, Kitwood went on to describe the principles of "person-centered care", which is characterized by: recognizing that the person with dementia is able to experience life and relationships, offering and respecting choices, incorporating the person's past life into their care, and focusing on the person's strengths rather than weaknesses. CST incorporates these elements of person-centered care into sessions, guided by a set of key principles, which have been adapted for the iCST program. iCST activities are inherently person centered, as they are delivered in a one-to-one setting, thus can be completely personalized. Furthermore delivery by a familiar carer could be beneficial as they have a unique insight into the person's life history, interests, and abilities amassed over a long period of time.

\section{Preliminary consultations with service users and health care professionals Design (preclinical phase)}

Prior to designing the iCST program and drafting the materials, preliminary consultations with service users and health care professionals took place and a panel of experts was invited to advise the research team. Service-user involvement can help develop theoretically coherent and evidence-based interventions, which are more likely to be meaningful and address the needs of the target population. ${ }^{29}$ In preparation for the development of the program, a literature-scoping exercise was also performed to determine the current understanding of the field and identify any potential for innovation. ${ }^{30}$

\section{Sample}

Twenty-seven care staff and 20 carers and people with dementia participated in the consultations. Care staff were approached for their views at CST training days, and carers and people with dementia were contacted through the charity Dementia UK. The advice panel was made up of two carers and two professionals.

\section{Methods}

The consultations focused on the acceptability of an individualized version of CST. Participants were invited to discuss their ideas, needs for the program, and the feasibility of developing the program. Alongside these discussions the research team examined the current literature on group CST, including the $\mathrm{CST}^{31}$ and maintenance CST manuals, ${ }^{32}$ and one-to-one programs of cognitive stimulation and reality orientation. This evidence was also reviewed by the panel, which advised the research team about the adaptation of the group CST and individual approaches identified.

\section{Results}

\section{First draft of iCST materials (Draft I)}

Acceptability of iCST program

Carers and people with dementia felt that an individualized version of CST would be very useful and priority should be 
placed on its development. Participants anticipated that the program would be beneficial in a variety of ways including by bringing the carer and person with dementia closer together, providing those who are unable to get out of the house an opportunity to take part in CST, and possibly using the program as an alternative if medication is unsuitable for the person with dementia.

\section{Structure and duration of iCST sessions}

A key feature of the group CST sessions is their consistent structure, which comprises introductions and a warm-up activity (eg, group song, softball game, discussion of orientation information), a themed mentally stimulating activity, and session closing/summary. As the iCST sessions are intended to be delivered by a family member or friend, the formal "introduction" element of the session was deemed unnecessary and omitted, as was the "closing of the session". However, iCST sessions include the discussion of orientation information (eg, date, time, weather), current affairs, and a themed activity. Thus the iCST session structure represents a simplified version of the original CST model.

iCST sessions last 20-30 minutes, making them shorter than the 45-minute session duration recommended in the group CST programs. Participants of the discussion forum felt that sessions should not be too long. Onder et al's study suggested this 20-30 minute duration was feasible. ${ }^{17}$ It is unclear whether there is an optimum "dose" of CST. However, it was reasoned that group participants receive 90 minutes of CST per week and experience benefits in cognition and QoL, thus iCST participants may experience similar benefits if given the opportunity to spend an equal amount of time taking part in activities. As a result of the reduction in session duration, the 38 group-CST sessions were each split into two iCST sessions, with the exception of the final session, resulting in a 75 -session program.

\section{Content of the iCST program}

The panel of professionals and carers advised that the iCST manual should be more concise than the group manuals and the instructions provided should be simple and free from "academic terminology". It was also suggested that the dyadic nature of the program should be emphasized throughout. The iCST session themes (eg, "My life", "Food", "Current affairs") and many of the ideas for activities were taken directly from the group CST manuals. The team also had access to a bank of resources that had been created by researchers for use in groups in the maintenance cognitive stimulation therapy (CST) for dementia trial. Activities were reviewed according to their scope for adaptation for a one-to-one session, and how well they had been received by group members in the trial. Those that had received positive responses and appeared relevant for delivery in a one-to-one setting were incorporated into the first draft of the iCST manual. The consultees felt that the activities should be varied so that there would be flexibility to cater for the abilities of the person with dementia.

Neither of the group CST manuals ${ }^{31,32}$ supplies paperbased resources for the suggested activities outlined. The group CST programs are designed to be delivered by staff members in day centers or residential care facilities, thus it is expected resources may be available to them, or can be sourced with support from their workplace. However, the decision was made to provide preprepared materials for iCST because it was acknowledged that family carers may have difficulty in acquiring materials themselves, or may be unable to take the time to do so.

\section{Principles of the iCST program}

The guiding principles of the group CST programs were adapted to create the nine key principles of iCST. Many of the principles developed as part of the original program are applicable in a one-to-one setting, and all are founded on the person-centered approach to care. However, those specific to a group environment were omitted (eg, "inclusion" and "involvement"). The advice panel recommended that the principles should be concise for ease of understanding.

\section{Design and format of iCST manual Draft I}

A graphic designer from the University College London (UCL) Creative Media Services department developed the layout of the first draft of the manual. Key requirements expressed by the expert panel were that the manual should be visually appealing with a simple and clear layout, taking a similar approach to the group CST manuals. The design features of the manual were applied in the first drafts of the activity workbook by the research team in house.

\section{Stage 2: evaluation of Draft I (Phase I: modeling process) \\ Design}

As recommended in the MRC guidelines, ${ }^{1}$ the modeling phase of the development of the iCST intervention included focus groups and interviews. The first drafts of the iCST manuals and activity workbooks, and prototype toolkit items were presented to carers and people with dementia for appraisal. 


\section{Sample}

Twenty-four carers and 28 people with dementia participated in the focus groups and interviews. Participants were recruited from the voluntary sector, memory services, and a local authority organization.

\section{Method}

Ten individual interviews and six focus groups (three with people with dementia, two with carers, and one with carers and their relative with dementia) were carried out. The purpose of combining these qualitative methods was to obtain data with both depth and breadth. ${ }^{33}$ The groups and interviews involved discussion of mental stimulation and mentally stimulating activities, consideration of the feasibility of the iCST intervention and exploration of potential barriers that might be encountered during the program, and appraisal of the iCST materials. In addition, people with dementia were invited to try a selection of the iCST activities and provide feedback about their enjoyment and the level of difficulty of the activities. Materials for the first twelve sessions of the program were presented in the groups and interviews.

\section{Analysis}

Audio recordings of the groups and interviews were transcribed by a medical transcription service, and inductive thematic analysis techniques applied to the data. ${ }^{34}$ The results from the groups and interviews were considered at first separately, then compared and grouped by source (carer and person with dementia).

\section{Results}

The feedback gathered from the groups and interviews was used alongside the findings from the field-testing phase to create the second drafts of the iCST manuals, activity workbooks, and toolkit (see the "Results" subsection of "Stage 3: Field-testing Draft 1 (Phase II: piloting)" and Figure 1).

\section{Stage 3: field-testing Draft I (Phase II: piloting) \\ Design}

The data from the focus groups and interviews were restricted in that participants could only discuss the program "in theory", and only materials for the first twelve sessions were available at this stage. Owing to time constraints, the program was not tested in full ( 75 sessions over 25 weeks) by any one dyad; rather it was split into six sections, and each dyad was allocated $12-15$ sessions to complete. Field testing is worthwhile prior to a main RCT as issues with the research design or intervention can be identified and resolved before investing time, resources, and funding in a full study. ${ }^{35}$

\section{Sample}

Twenty-two carers and people with dementia participated in the field-testing. The sample of carers consisted of both family members $(n=16)$ and paid carers $(n=6)$. The research team liaised with key contacts from the voluntary sector, the NHS, and local authority organizations established during recruitment for the focus groups and interviews to recruit family carers. Five paid carers were recruited from a private home-care agency in North London, and a live-in carer approached the team about participating after seeing an article about the study in an Age Concern newsletter.

\section{Methods}

Dyads completed a portion of the program with training and support from a researcher. "Monitoring progress" forms were used to gather data about each activity, including quantitative ratings of enjoyment, interest, communication, and level of difficulty. Detailed qualitative feedback was gathered during the setup visit, telephone support calls with researchers, and debrief visits.

\section{Results}

Consistent with the feedback from the focus groups and interviews, carers felt the manual and activity workbook were clearly laid out and written in a way that was easy to understand. Both carers and people with dementia commented on how visually appealing they found the materials, notably the quality of the images used in the activity workbooks and the clear layout and professional look of the manuals.

\section{Modifications incorporated into Draft 2}

The feedback from the modeling activities and field-testing was consolidated to create second drafts of the iCST materials, which were professionally printed prior to the launch of the online survey (see "Stage 4: Online survey and consensus conference"). Minor changes to the manuals included the correction of some mistakes in spelling and grammar, editorial changes to improve the clarity of some of the instructions provided, and alterations to the size of some text and images. The monitoring-progress forms underwent significant adjustments in response to feedback from carers who felt the approach to appraising sessions should be more 
informal to avoid the person with dementia feeling as though their performance is being scrutinized. "Monitoring progress" was replaced with "How was your session today?", which invites a more collaborative approach to session appraisal. In addition, carers felt that it would be too time-consuming to assess every session, so feedback was sought every two sessions, and grouped by theme instead. The rating scale was also amended to discourage bias toward rating at the midpoint of the scale.

\section{Practical issues with intervention delivery}

Few difficulties were experienced with the program itself. However, challenges related to the program structure and technique were reported in a small number of cases. Some carers struggled with the orientation discussion at the beginning of each session; others reported finding delivering the program "hard", struggling to apply the key principles, and having difficulty maintaining conversation. In terms of delivery, the main barriers to completing sessions were lack of time and illness of the carer or person with dementia. The materials were not changed in response to these issues at Draft 2 stage, but were considered as part of the consensus process (see the "Results" subsection under "Stage 4: Online survey and consensus conference"), and the findings provided justification for the amendment of the guidance included the final draft.

\section{Stage 4: online survey and consensus conference Design}

A two-round modified Delphi process was conducted. The first round was an online survey and the second a conference. The Delphi technique was selected as a means of achieving consensus on themes that participants had been unable to reach agreement on in the focus groups, interviews, and field testing. Delphi participants can be valuable contributors to decision-making processes, informed by their direct knowledge and experience. ${ }^{36}$

\section{Sample}

Twenty-five people completed the online survey and 28 attended the conference. Sixteen participants (57\%) completed the Delphi process by taking part in both rounds. The sample consisted of a variety of professionals and service users including academics, health care professionals, and carers.

\section{Methods}

Participants were sent a copy of one of the six serialized manuals and activity workbooks in the post along with instructions for the online survey. Consent was obtained as part of the survey. A conference was subsequently held at UCL. Attendees were presented with the findings of the focus groups, individual interviews, and field testing, then asked to work in small groups on six key themes: the iCST toolkit, getting started with iCST, home-based training for carers, sessions associated with difficulties in field testing, the presentation of iCST, and support for carers delivering iCST.

\section{Results}

Final version of the iCST materials (main RCT)

The final version of the iCST materials was produced based on the findings of the Delphi process. This draft was printed and bound professionally for use in the main RCT.

\section{Modifications incorporated into the final version}

The online-survey respondents felt that the manual and key principles should be more person centered and focused on the positive outcomes of taking part in the sessions together. Terminology in the manual considered to be "too academic" was rephrased in accordance with the feedback that the manual should be easy to understand. Additionally, the introduction was made more concise in an effort to add clarity to the information presented. Another suggestion was that there needed to be a clearer distinction between level $\mathrm{A}$ and level $\mathrm{B}$ activities.

The contents of the iCST toolkit were reviewed at the conference. The consensus group concluded that the physical games materials provided should be adequate for use indoors as well as outdoors, to cater for those with limited mobility or a lack of outdoor access. The UK map included in the second version of the toolkit was replaced with a map showing counties, which was thought to be more useful than one showing just towns and cities. A set of marbles was considered a potential health and safety risk, so was not included in the final toolkit.

Field-testing participants felt that more guidance about the warm-up elements of the session (eg, discussion of date, time, weather) would be helpful. Additional information on this was not incorporated into the second draft of the manual. However, the need to include extra information was also highlighted by the online survey and conference participants, so a "Getting started" section was developed and included in the final version of the manual.

Sessions that had been poorly rated in the field testing and were thought to be too challenging by the consensus groups and online-survey respondents were simplified. These included "food" and "orientation" activities. Additionally, it 
was suggested that some of the stimuli (eg, images, topics) were not relevant to the age group of the people likely to participate in the program. Alternative images and suggestions for activities were sourced in response.

At the final consensus meeting there was still some debate around the format in which the manual and workbook should be presented (ie, as one document or serialized). However, carers felt that the whole program should appear in one manual with one accompanying activity workbook, and as a result this format was adopted for the final version.

\section{Discussion}

The stages specified in the MRC guidelines were implemented in the development of the iCST intervention. ${ }^{1}$ The first step was to identify and review the evidence base for group $\mathrm{CST}^{3}$ and one-to-one cognitive stimulation programs. ${ }^{15-17}$ Subsequently, a theoretical understanding of the likely process of change in the outcomes observed in previous research (eg, cognition and QoL for the person with dementia, and wellbeing of the carer). The development of the first version of the iCST materials was guided by the evidence gathered and reviewed in these preliminary stages. The intervention was progressively refined in a series of qualitative evaluations that comprised focus groups, interviews, a consensus survey and conference, and a field-testing phase.

An advantage of such a rigorous development process is that the intervention and program materials have been developed to the point at which they can be reasonably expected to have a worthwhile effect when examined in a full-scale trial. This is recommended by the MRC as a means of safeguarding against problems of acceptability, compliance, delivery of the intervention, recruitment and retention, and smaller-than-expected effect sizes, which can undermine the evaluation of an intervention. ${ }^{1}$ Thorough development, including a field-testing or piloting phase, can also prevent unwarranted full-scale evaluation, which can be costly and time-consuming. Service-user involvement in clinical research trials is recommended by the UK Department of Health. ${ }^{37}$ The focus groups, interviews, and field testing provided a platform by which people with dementia and carers could indicate their views about, requirements of, and expectations of iCST. Drawing on the experiences of individuals who are "experts" in their knowledge of dementia and mental health services can be a useful way of improving care packages and services, ensuring these are appropriately tailored and fit for purpose. ${ }^{38}$

A feature of the Delphi consensus process is the collection of feedback in multiple stages from the panel of experts taking part, which carries the risk of a low response rate and can compromise the quality of information obtained. ${ }^{39}$ However, the risk was reduced in this study as the Delphi process comprised only two stages. Participant retention rate was relatively high across the two stages (57\%). Consensus was achieved on all presented themes with the exception of how the manual should be presented (ie, serialized vs complete manual).

Whilst the implementation of the MRC framework and the careful development of an intervention represent best practice, this process does not guarantee either the efficacy of the intervention or that the full-scale evaluation will be unaffected by any challenges in the design, methods, and implementation. No formal measures of our outcomes of interest (eg, cognition and QoL for the person with dementia) were taken during the field-testing phase, providing no indication of the likely efficacy of the intervention. However, some carers reported improvements in the communication skills and alertness of the person as well as enjoyment. In addition, some dyads felt that participating in iCST had improved their understanding of the person and, as a result, their relationship with them. A large-scale Phase III RCT is required to provide more definitive evidence of the effectiveness of the intervention. If the findings of the main RCT are clinically significant, the data obtained from each phase in the process of developing the intervention may add to the understanding of the mechanisms underpinning the effects of the intervention. However, if the intervention does not succeed, the thorough nature of the development phase may yield some insight into the possible reasons for this.

\section{Conclusion}

The development phase of the iCST program was extensive, resulting in the production of two drafts and a final version of the iCST manual, activity workbook, and toolkit. Feedback and advice were gathered from experts in the field and service users throughout the process to ensure the program was tailored to the needs of people with dementia and their carers. The next step in the process of the development of this complex intervention (Phase III) is to evaluate the final version of the program in a large-scale multicenter RCT.

\section{Acknowledgments}

This article presents independent research supported and funded by the National Insititute for Health Research (NIHR) under the Health Technology Assessment (HTA) program. The grant holders are Professors Orrell (UCL), Burns (Manchester), Russell (Swansea), Woods (Bangor), Moniz-Cook 
(Hull), Knapp (London School of Economics), and Dr Spector (UCL). The views expressed in this article are those of the authors and not necessarily those of the NHS, the NIHR, or the Department of Health.

We also acknowledge the involvement of the following organizations in the recruitment for, and organization of, research activities: Carers of Lewisham, Jewish Care, Crossroads, Dementia UK, Staywell, and Living Well Resource Centre.

\section{Authors' contributions}

LAY prepared the manuscript based on a chapter of her $\mathrm{PhD}$ thesis. LAY recruited participants, facilitated focus groups, conducted individual interviews, and contributed to the analysis of the data gathered. PL organized the online survey and consensus conference, and contributed to data analysis. LAY, PL, VO, AS, and MO contributed to the development of the iCST approach. All authors participated in the consensus conference. All authors reviewed and commented on drafts of the manuscript, revising it critically for important intellectual content, and read and approved the final manuscript for publication.

\section{Disclosure}

The authors declare no conflicts of interest in this work.

\section{References}

1. Craig P, Dieppe P, Macintyre S, Michie S, Nazareth I, Petticrew M; Medical Research Council Guidance. Developing and evaluating complex interventions: the new Medical Research Council guidance. BMJ. 2008;337:a1655.

2. Aguirre E, Spector A, Hoe J, et al. Development of an evidence based extended programme of Maintenance Cognitive Stimulation Therapy (CST) for people with dementia. Nonpharmacol Ther Dement. 2011; 1(3):197-215.

3. Spector A, Thorgrimsen L, Woods B, et al. Efficacy of an evidencebased cognitive stimulation therapy programme for people with dementia: randomised controlled trial. Br J Psychiatry. 2003;183:248-254.

4. Woods B, Aguirre E, Spector AE, Orrell M. Cognitive stimulation to improve cognitive functioning in people with dementia. Cochrane Database Syst Rev. 2012;2:CD005562.

5. Knapp M, Thorgrimsen L, Patel A, et al. Cognitive stimulation therapy for people with dementia: cost-effectiveness analysis. Br J Psychiatry. 2006;188(6):574-580.

6. NHS Institute for Innovation and Improvement. An Economic Evaluation of Alternatives to Antipsychotic Drugs for Individuals Living with Dementia. Coventry: NHS Institute for Innovation and Improvement; 2011. Available from: http://www.institute.nhs.uk/images//Call_to_ Action/20\%2010\%202011\%20An\%20economic\%20evaluation \%20 of $\% 20$ alternatives $\% 20$ to $\% 20$ antipsychotic $\% 20$ drugs $\% 20$ for $\% 20$ individuals $\% 20$ living $\% 20$ with $\% 20$ dementia $\% 20 \% 20$ Final $\% 20$ Report.pdf. Accessed July 10, 2014.

7. National Institute for Health and Clinical Excellence and the Social Care Institute for Excellence (NICE-SCIE): Dementia: supporting people with dementia and their carers in health and social care. Clinical Guideline 42. London: NICE-SCIE; 2006.
8. Prince M, Bryce R, Ferri C. World Alzheimer Report: The benefits of early diagnosis and intervention. London: Alzheimer's Disease International; 2011. Available from: http:/www.alz.co.uk/research/ WorldAlzheimerReport2011.pdf. Accessed November 6, 2014.

9. Orrell M, Aguirre E, Spector A, et al. Maintenance cognitive stimulation therapy for dementia: single-blind, multicentre, pragmatic randomised controlled trial. Br J Psychiatry. 2014;204(6):454-461.

10. Streater A, Spector A, Aguirre E, et al. Maintenance Cognitive Stimulation Therapy (CST) in practice: study protocol for a randomized controlled trial. Trials. 2012;13(1):91.

11. Mahmood S, Ahmed S, Orrell M, Kinsler H. Developing cognitive stimulation therapy (CST) for dementia with South Asian ethnic groups. In: Acosta D, Ketteringham A, Ballard C, editors. 27th International Conference of Alzheimer's Disease International [conference proceedings], London, UK, March 7-10, 2012. Bologna: Medimond; 2012: 57-60.

12. Yamanaka K, Kawano Y, Noguchi D, et al. Effects of cognitive stimulation therapy Japanese version (CST-J) for people with dementia: a single-blind, controlled clinical trial. Aging Ment Health. 2013;17(5): 579-586.

13. Aguirre E, Spector A, Orrell M. Guidelines for adapting cognitive stimulation therapy to other cultures. Clin Interv Aging. 2014;9:1003-1007.

14. Spector A, Gardner C, Orrell M. The impact of Cognitive Stimulation Therapy groups on people with dementia: views from participants, their carers and group facilitators. Aging Ment Health. 2011;15(8): 945-949.

15. Moniz-Cook E, Agar S, Gibson G, Win T, Wang M. A preliminary study of the effects of early intervention with people with dementia and their families in a memory clinic. Aging Ment Health. 1998;2(3):199-211.

16. Quayhagen MP, Quayhagen M. Testing of a cognitive stimulation intervention for dementia caregiving dyads. Neuropsychol Rehabil. 2001;11(3-4):319-332.

17. Onder G, Zanetti O, Giacobini E, et al. Reality orientation therapy combined with cholinesterase inhibitors in Alzheimer's disease: randomised controlled trial. Br J Psychiatry. 2005;187:450-455.

18. Spector A, Orrell M. Using a biopsychosocial model of dementia as a tool to guide clinical practice. Int Psychogeriatr. 2010;22(06):957-965.

19. Albert MS. Changes in cognition. Neurobiol Aging. 2011;32 Suppl 1: 58-63.

20. Katzman R. Education and the prevalence of dementia and Alzheimer's disease. Neurology. 1993;43(1):13-20.

21. Hall L, Orrell M, Stott J, Spector A. Cognitive stimulation therapy (CST): neuropsychological mechanisms of change. Int Psychogeriatr. 2013;25(03):479-489.

22. Swaab DF, Dubelaar EJ, Hofman MA, Scherder EJ, Van Someren EJ, Verwer RW. Brain aging and Alzheimer's disease; use it or lose it. Prog Brain Res. 2002;138:343-373.

23. Béland F, Zunzunegui MV, Alvarado B, Otero A, del Ser T. Trajectories of cognitive decline and social relations. J Gerontol B Psychol Sci Soc Sci. 2005;60(6):320-330.

24. Yeh SC, Liu YY. Influence of social support on cognitive function in the elderly. BMC Health Serv Res. 2003;3(1):9.

25. Elwood PC, Gallacher JE, Hopkinson CA, et al. Smoking, drinking, and other life style factors and cognitive function in men in the Caerphilly cohort. J Epidemiol Community Health. 1999;53(1):9-14.

26. Moyle W, Kellett U, Ballantyne A, Gracia N. Dementia and loneliness: an Australian perspective. J Clin Nurs. 2011;20(9-10):1445-1453.

27. Woods B, Thorgrimsen L, Spector A, Royan L, Orrell M. Improved quality of life and cognitive stimulation therapy in dementia. Aging Ment Health. 2006;10(3):219-226.

28. Kitwood T. Dementia Reconsidered: The Person Comes First Buckingham and Philadelphia, PA: Open University Press; 1997.

29. Burnell KJ, Selwood A, Sullivan T, Charlesworth GM, Poland F, Orrell M. Involving service users in the development of the Support at Home: Interventions to Enhance Life in Dementia Carer Supporter Programme for family carers of people with dementia. Health Expect. 2012. Epub October 18. 
30. Erich K, Freeman G, Richards S, Robinson I, Shepperd S. How to do a scoping exercise: continuity of care. Research Policy and Planning. 2002;20(1):25-29.

31. Spector A, Thorgrimsen L, Woods RT, Orrell M. Making a Difference: An Evidence-Based Group Programme to Offer Cognitive Stimulation Therapy (CST) to People with Dementia. London: Hawker; 2006.

32. Aguirre E, Spector A, Streater A, Hoe J, Woods B, Orrell M. Making a Difference 2: An Evidence Based Group Programme to Offer Maintenance Cognitive Stimulation Therapy (CST) to People with Dementia. London: Hawker; 2011.

33. Lambert SD, Loiselle CG. Combining individual interviews and focus groups to enhance data richness. J Adv Nurs. 2008;62(2):228-237.

34. Boyatzis RE. Transforming Qualitative Information: Thematic Analysis and Code Development. Thousand Oaks, CA, London, and New Delhi: Sage; 1998.
35. Mason DJ, Zuercher SL. Pilot studies in clinical nursing research. $J N Y$ State Nurses Assoc. 1995;26(2):11-13.

36. Murphy MK, Black N, Lamping DL, et al. Consensus development methods and their use in clinical guideline development. Health Technol Assess. 1998;2(3):i-iv, 1-88.

37. NHS Executive. The NHS Plan: A Plan for Investment, a Plan for Reform. London: Department of Health; 2000. Available from: http:/www.dh.gov.uk/prod_consum_dh/groups/dh_digitalassets/@ dh/@en/@ps/documents/digitalasset/dh_118522.pdf. Accessed November 6, 2014.

38. Tait L, Lester H. Encouraging user involvement in mental health services. Adv Psychiatr Treat. 2005;11(3):168-175.

39. Sandford BA, Hsu CC. The Delphi technique: making sense of consensus. Practical Assessment, Research and Evaluation. 2007; 12(10):1-8.
Clinical Interventions in Aging

\section{Publish your work in this journal}

Clinical Interventions in Aging is an international, peer-reviewed journal focusing on evidence-based reports on the value or lack thereof of treatments intended to prevent or delay the onset of maladaptive correlates of aging in human beings. This journal is indexed on PubMed Central, MedLine,

\section{Dovepress}

CAS, Scopus and the Elsevier Bibliographic databases. The manuscript management system is completely online and includes a very quick and fair peer-review system, which is all easy to use. Visit http://www.dovepress. com/testimonials.php to read real quotes from published authors. 